\title{
Fluoxetine reduced impulsive aggressive behaviour in patients with a personality disorder
}

\author{
Coccaro EF, Kavoussi RJ. Fluoxetine and impulsive aggressive behavior in personality-disordered subjects. Arch Gen Psychiatry 1997 \\ Dec;54:1081-8.
}

\section{Question}

In patients with a personality disorder, can fluoxetine hydrochloride reduce impulsive aggressive behaviour?

\section{Design}

Randomised, double blind, placebo controlled trial with 3 months follow up.

\section{Setting}

University psychiatry department in Pennsylvania, USA.

\section{Patients}

40 patients (mean age 38 y, 70\% men), recruited from an outpatient population or by self referral, who had a DSM-III-R diagnosis of personality disorder with a current history of impulsive aggressive behaviour and irritability. Exclusion criteria were mania or hypomania; schizophrenia or delusional disorder; current major depression; or dependence on alcohol or drugs.

\section{Intervention}

All patients participated in a 2 week placebo lead-in phase. 27 patients were then allocated to oral fluoxetine hydrochloride, 20 $\mathrm{mg} /$ day for the first 4 weeks which could then be increased to 40 $\mathrm{mg} /$ day if the mean score on the aggressive subscale of the Overt Aggressive Scale-Modified for Outpatients (OAS-M) for the previous 2 weeks had not decreased to $<25 \%$ of the mean score recorded during the lead-in phase. After 8 weeks the dose could again be increased to $60 \mathrm{mg} /$ day for the same reason as indicated above. 13 patients were allocated to placebo.

\section{Main outcome measures}

The main outcome measures were change in scores on the aggressive and irritability subscales of the OAS-M, and Clinical Global Impression Rating of Improvement (CGI-I).

\section{Main results}

$52 \%$ of patients in the fluoxetine group and $69 \%$ in the placebo group completed the study. Compared with patients in the placebo group, patients in the fluoxetine group had lower scores on the aggressive subscale of the OAS-M at week $12(\mathrm{p}=0.01)$, and at endpoint (ie, intention to treat analysis with the last visit carried forward, $p=0.02$ ). Similarly, patients in the fluoxetine group had lower scores on the irritability subscale of the OAS-M at week $6(\mathrm{p}=0.02)$, week $12(\mathrm{p}=0.01)$, and at endpoint $(p=0.006)$. More patients in the fluoxetine group were responders on the CGI-I (defined as very much improved or much improved) than those in the placebo group (at endpoint, $\mathrm{p}=0.001$ ) (table). Results were not influenced by secondary measures of depression, anxiety, or alcohol use.

\section{Conclusion}

Fluoxetine reduced impulsive aggressive behaviour in patients with a personality disorder who had prominent histories of impulsive aggressive behaviour.

Fluoxetine v placebo at endpoint*

Outcome Fluoxetine Placebo RBI $(95 \%$ CI $) \quad$ NNT (CI)

\begin{tabular}{lllll}
\hline Responders on the CGI-I & $67 \%$ & $23 \%$ & $189 \%(23$ to 736$)$ & 3 (1 to 9$)$
\end{tabular}

*CGI-I = Clinical Global Impression Rating of Improvement. Other abbreviations defined in glossary; RBI, NNT, and CI calculated from data in article.

Sources of funding: National Institute of Mental Health and Lilly Research Laboratories, Indianapolis.

For correspondence: Dr E F Coccaro, Clinical Neuroscience Research Unit, Department of Psychiatry, MCP Hahnemann School of Medicine, Allegheny University of the Health Sciences, 3200 Henry Avenue, Philadelphia, PA 19129, USA. Fax +1 2158424321 .

\section{Commentary}

Given the well established link between low central serotonin function and impulsive and aggressive acts, it is entirely feasible that serotonergic drugs should lessen this behaviour. ${ }^{2}$ Because reducing aggressive behaviour is one of the major challenges for mental health services, clinicians should welcome this carefully designed and conducted trial by Coccaro and Kavoussi. It provides, however, only partial support for the clinical effectiveness of fluoxetine in personality disordered subjects with impulsive aggressive behaviour. This is the case because the benefits in the active drug group were primarily verbal aggression and aggression against objects, not physical aggression against other people (for which a much longer trial would be required because of its relative infrequency), nor self perceived aggression and impulsivity. Lack of any initial increase in irritability or aggression in patients receiving fluoxetine is interesting in view of previous concerns that it might increase this behaviour.

The exclusion from the trial, for understandable reasons, of patients with depression or substance abuse limits the extent of applicability of these results for clinical practice because depression and substance abuse are common features in patients with personality disorder and impulsive aggressive behaviour. Compliance is another important determinant of clinical applicability. The fact that half of the fluoxetine treated subjects had dropped out of the study by week 12 shows the considerable challenge of keeping patients on this type of medication. As the authors themselves suggest, longer term treatment may be required to see the full benefits, and maximisation of these benefits may require a combination of psychological treatment such as cognitive behaviour therapy with drug treatment-a proposal that clearly warrants evaluation.

The present study represents an encouraging step in finding ways of reducing impulsive aggressive behaviour in patients with personality disorder and one that offers clinicians a therapeutic strategy worth trying.

Keith Hawton, DM, FRCPsych University of Oxford Oxford, UK

1 Brown GL, Goodwin FK, Ballenger JC, et al. Psychiatry Res 1979;1:131-9.

2 Coccaro EF, Slever LJ, Klar H, et al. Arch Gen Psychiatry 1989;46:587-99. 